number of factors ${ }^{10}$. This holds for assortative mating when the number of factors is small. There is no reason to believe that the situation is reversed when the number of factors is large. Fisher ${ }^{2.9}$, himself, believed this to be the case for his model and I agree with him. For, in Fisher's model, where add., dev., dom. and cov. stand for additive, deviation, dominance and covariance respectively, sib covariance - - cov.(add. dev., add. dev.) : cov.(add. dev., dom. dev.) cov.(dom. dev., dom. dev.), and parent-child covariance $=$ cov. (add. dev., add. dev.) : cov.(add. dev., dom. dev.). All covariances on the right of these equations are positive. Mendelian segregation will ensure that cov.(add.dev., add. dev.) and cov.(add. dev., dom. dev.) in the two equations are equal. It is obvious that in presence of dominance, sib covariance will be greater than the parent-child covariance. In absence of dominance they will be equal. Incidentally, I do not assert that it is 'impossible' for parent-child correlation to exceed the sib correlation, only that, in Fisher's model, it should not.

Atam VetTa

Oxford Polytechnic,

Oxford, UK

1 Vetta, A. Nature 263, 316 (1976)

2 Fisher, R. A. Trans. R. Soc. Edinb, 52, 399 (1918).

Bulmer, M. G. Nature 266, 195 (1977)

O'Ferrall, G. M. \& Cunningham, E. P. Lives. Prod. Sc, 1, 87 (1974)

5 Wright, S. in Quantitative Inheritance (eds. Reeve, E.C.R. \& Waddington, C. H.) (HMSO, London, 1952)

Corw, J. F. \& Kimura, M. An Introduction to Population Genetic Theory

N C C P \& London, 1971). University of Chicago Press, 1955).

8 Fisher, R. A. Proc. R. Soc. Edinb. 42, 321 (1922).

Fisher, R. A. Proc. R. Soc. Edinb. 42, 321 (1922). Selection (Dover, New York, 1968).

to Falconer, D. S. An Introduction to Quantitative Genetics (Oliver and Boyd, Edinburgh, 1960).

\section{Cholecystokinin produces bait shyness in rats}

BECAUSE an injection of cholecystokinin (CCK) (or of the synthetic C-terminal octapeptide of $\mathrm{CCK}^{1}$ ) reduces food intake in the fasted rat, it has been suggested by Gibbs, Smith and colleagues ${ }^{2-6}$ that CCK may be a satiety signal. We present here evidence against this conclusion.

To quote from ref 4 , "In order to establish CCK as a physiological satiety signal, one requirement is that CCK does not inhibit feeding by inducing nausea or other subclinical sickness". An attempt to detect such sickness was made by injecting CCK after the rats had drunk a saccharin solution. If sickness occurs after CCK then less of the saccharin solution should be drunk on a subsequent occasion ${ }^{7,8}$.

The tests for nausea already performed ${ }^{2,3}$, however, were quite insensitive. In the first ${ }^{2}$ the rats were extremely thirsty $\left(23 \frac{1}{2} \mathrm{~h}\right)$ and were given a very attractive sweet taste to drink $(0.25 \%$ saccharin). Such test conditions would only be expected to reveal a very strong nausea induced by CCK. The dose of CCK used in this nausea test suppressed eating by only $50 \%$ when the CCK was injected before the rat ate ${ }^{2}$. This suggests that only a mild degree of nausea was induced by that dose of CCK if nausea was indeed the explanation. The results of the test showed no effect of CCK $\left(40 \mathrm{U} \mathrm{kg}^{-1}\right)$ or synthetic terminal octapeptide of CCK $\left(40 \mathrm{U} \mathrm{kg}^{-1}\right)$ but a strong effect of $0.15 \mathrm{M}$ lithium chloride as $0.6 \%$ body weight. Lithium chloride in this dosage is a very powerful aversive agent. Even higher levels of thirst were used in a comparison of apomorphine, another strong aversive agent, with CCK (40 $\mathrm{U} \mathrm{kg}^{-1}$ ) (ref. 4). Conditioned aversion was shown to apomorphine but not to CCK. Whilst these studies show that in the doses used, CCK (or the C-terminal octapeptide) is less aversive than $\mathrm{LiCl}$ or apomorphine, more sensitive tests of nausea might reveal intermediate degrees of aversiveness.

In our first experiment we used rats (Charles River, male Sprague-Dawley) 22-h thirsty and given 8-min exposure to a novel neutral flavour (almond Schilling $0.5 \%$ in water). Immediately after this drinking period nine rats were injected intraperitoneally with 40 Ivy dog units per $\mathrm{kg}$ of the terminal octapeptide in saline ${ }^{9}$, and $8 \mathrm{~h}$ later the rats were given water ad libitum for $2 \mathrm{~d}$. Then they were again water deprived for $22 \mathrm{~h}$. Both groups were then again presented with the almond solution for $8 \mathrm{~min}$. The mean amount consumed on the first day of exposure was almost identical for the experimental rats $(10.3 \mathrm{ml})$ and for the control rats $(10.4 \mathrm{ml})$, but on the second presentation the control rats drank a mean of $14 \mathrm{ml}$ as against $9.6 \mathrm{ml}$ consumed by experimental animals $(t=3.66$, d.f. $=16$, $P<0.01$ ). We used an even more sensitive indicator of conditioned aversion in our second experiment by using a preference instead of a single bottle method as a test. A group of 14 naïve Sprague-Dawley rats underwent $22 \mathrm{~h}$ water deprivation; half the rats were then presented with almond flavoured water and the others with banana flavoured water (both $0.5 \%$ Schilling). After $24 \mathrm{~h}$ of water ad libitum the rats were again deprived of water for $22 \mathrm{~h}$ and allowed to drink the alternate flavour. Immediately following the consumption of this flavour, the experimental rats were injected with CCK octapeptide as above, while the control rats were injected with vehicle. After $24 \mathrm{~h}$ of water ad libitum, rats were again fluid deprived for $22 \mathrm{~h}$. They were then given a 30 -min simultaneous choice between the two flavoured solutions. The controls drank a mean of $8.9 \mathrm{ml}$ of the flavour presented on the first day, and a mean of $8.6 \mathrm{ml}$ of the fluid presented on the second day when these fluids were simultaneously presented. In contrast, the rats injected with the CCK C terminal octapeptide drank a mean of $9.4 \mathrm{ml}$ of the flavour that had been presented on the first day, but only a mean of $3.3 \mathrm{ml}$ of the flavour paired with the octapeptide when the two flavours were presented simultaneously. There was a significant difference between the experimental and control groups ( $t=4.36$, d.f. $=12, P<0.001)$, indicating a larger conditioned aversion due to the octapeptide measured by this more sensitive method. We therefore conclude that the $\mathrm{C}$ terminal octapeptide of CCK in the dose administered to produce apparent satiation causes some form of sickness in the rat. Whether CCK is a satiation hormone is therefore left in considerable doubt.

We thank Dr A. Ondetti, Squibb Institute for Medical Research, for CCK octapeptide.

\section{J. A. Deutsch}

W. T. HARDY

Department of Psychology,

University of California, San Diego, La Jolla, California 92093

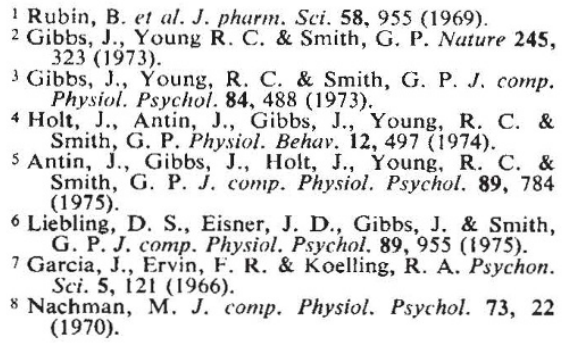

GibBs and SMith RePly-The production of a conditioned taste aversion, using a single dose of the synthetic octapeptide of CCK as the unconditioned stimulus, is not a critical test of the hypothesis that CCK mediates all or part of the satiety effect of food in the intestine. One indirect test of the hypothesis using the taste aversion paradigm would be the comparison of dose-response curves for both satiety and taste aversion. If the doses of CCK required for satiety were always equal to or larger than the doses that are aversive enough to produce a conditioned taste aversion, this would be indirect evidence against the hypothesis. Such a comparison has not been performed. The hypothesis will be most critical tested, however, when the concentration of circulating, endogenous CCK can be measured reliably during satiety. Then it should be possible to decide if ingested food releases sufficient CCK to account for the satiating effect of that ingested food.

\section{J. Gribs}

G. P. SMITH

University of Oxford, Department of Experimental Psychology,

South Parks Road,

Oxford, UK 